\title{
Ordering and reordering in the auditory and visual modalities
}

\author{
JANET METCALFE and DONALD SHARPE \\ University of British Columbia, Vancouver, British Columbia, Canada
}

\begin{abstract}
This study investigates the hypothesis that the modality effect (i.e., the often-found recall advantage of the last few items presented in the auditory-rather than visual-input modality) is attributable to a directional auditory trace. Such a directional trace should help performance of forward item-to-item recall, but should hurt performance if mental reordering is required. However, it was found in our Experiment 1 that mental item-to-item reordering of the materials did not affect the modality effect. In Experiment 2, strict control was exercised over the order of recall of chunks of items, as well as over the order of recall within chunks. It was found that the item-to-item reordering of the materials had little effect on the modality effect. However, the larger scale order of recall of the chunks had a large effect on the modality effect. If recall was in a forward order (i.e., first chunk, second chunk, third chunk), there was a dramatic superiority of the auditory materials on the last chunk. If the recall order was backward (third chunk, second chunk, first chunk), the modality effect was inconsequential. These results suggest that the modality effect is related to access to large memorial units rather than to itemto-item associations.
\end{abstract}

Many experiments investigating the role of input modality on human memory find that the last few items presented are better retained with auditory- rather than with visual-presentation modality (Craik, 1969; Crowder \& Morton, 1969; see Penney, 1975, for a review). The present experiments investigate the possibility that direction of recall is important for this effect. As Drewnowski (1980) noted, auditory input may result in an obligatory forward-directional trace. Such a trace could explain the modality effect because it might facilitate forward recall and perhaps have most impact on the recency items.

A number of experimental findings are consistent with the idea of a directional auditory trace. First, the modality effect is usually studied with forward-serial recall or with free recall, both types of recall for which a forward obligatory trace could improve performance. Second, Nilsson, Wright, and Murdock (1975) found that, in free recall, the order of output differs depending upon the input modality. With auditory input, subjects tend to recall the recency items first in a forward order, starting several items from the end of the list. With visual input, subjects tend to recall in a backward order, starting with the last

We wish to thank Elizabeth Bjork, Robert Bjork, and Eric Eich for helpful discussions related to this article. Judith Goldberg provided experimental assistance. We also wish to thank Emily Howard for her help in the presentation of some of the results from Experiment 1 to the Annual Convention of the American Psychological Association in Anaheim, CA, 1983. This research was facilitated by a Natural Sciences and Engineering Research Council of Canada (NSERC) postdoctoral fellowship, and by NSERC Grant A0505 to the first author. Requests for reprints should be sent to J. Metcalfe, Department of Psychology, University of British Columbia, Vancouver, British Columbia, Canada, V6T IW5. item of the list. This output-order performance might reflect differences in the directional format of the stored information. Third, Nilsson, Wright, and Murdock (1979) found that, when subjects were precued to recall in either a modified forward (auditory-like) order or a backward (visual-like) order, the extent of the modality effect was reduced, as compared to a control condition in which subjects were allowed to free recall. Although it is not obvious from the directional auditory trace hypothesis why the auditory-like recall order should help performance when the input had been visual, the decrement in the modality effect with backward recall fits nicely with the hypothesis. Fourth, whereas Madigan (1971) found the usual modality effect with forward-serial recall, the modality effect disappeared with backward-serial recall. Fifth, Experiment 3 by Nilsson (1979) showed that, when critical to-be-remembered words were presented successively in a list, a modality effect was obtained, but when the critical words were randomly scattered throughout the list, no modality effect was found. These results are consistent with a directional trace insofar as the cuing function of such a trace should be helpful only if the to-beremembered words are adjacent in memory, and may otherwise be harmful to performance.

Finally, Metcalfe, Glavanov, and Murdock (1981) have shown that, although auditory superiority results when subjects are required to recall in a temporal order within a chunk of words, visual superiority results when subjects are required to recall in a spatial order which, by the design of the experiment, is orthogonal to the temporal order. The most obvious interpretation of these results is that there is an affinity between auditory presentation and temporal recall, and an affinity between visual 
presentation and spatial recall. Although this interpretation is intuitively compelling, an equally viable interpretation of the results rests on the idea of a directional auditory trace. It may be that, in order to perform the spatial task, subjects had to mentally reorder the materials from the temporal order. If the auditory trace were intrinsically forward directional (and the visual trace were not), this reordering alone would be sufficient to produce the auditory deficit in the spatial case. This argument states that the spatial nature of the task in Metcalfe et al.'s (1981) study had little to do with the results. Rather, what may have been critical was the fact that subjects had to reorder the materials in one condition (resulting in poor auditory performance) and not in the other (resulting in a modality effect).

\section{EXPERIMENT 1}

Experiment 1 is similar to the experiments of Metcalfe et al. (1981), except that, instead of using spatial recall, subjects had to reorder the words within each chunk based on a nonspatial marker. Each subject was presented with lists of words, one at a time, that were divided into chunks of three words each. Within each chunk, there was a onesyllable, a two-syllable, and a three-syllable word. Subjects were asked either to recall the words in temporal order (TO) within each chunk, or to reorder the words so that recall was in the order one-, two-, and threesyllable word (RO). The order in which the items would have to be recalled in the RO condition was not predictable from the temporal order. Instead, if the traces were organized in an obligatory forward order, as the directional auditory trace hypothesis proposes, this organization would be expected to be disruptive in the RO condition. The specific prediction we made about this experiment was that the usual modality effect should be found in the TO condition, but should be reversed in the RO condition. The reason for expecting a reversal rather than no effect in the RO condition was that we thought the obligatory directional trace would actually interfere with the reorganization, rather than being neutral, as was expected in the visual case.

\section{Method}

Subjects. The participants in this experiment were 48 students in introductory psychology at the University of California, Los Angeles, who took part in the experiment for course credit.

Procedure. Subjects were told that they would be participating in a memory experiment in which they would be given a number of lists of words to study and remember. Each list consisted of nine common words. The lists were presented one at a time on index cards that were turned at a rate of one card per second. Each list of nine words was divided into three chunks, each made up of three words. A blank card (also presented at a 1-sec rate) separated one chunk from another. Within each chunk there was a one-syllable, a two-syllable, and a three-syllable word. Subjects were given booklets consisting of three rows, each with three blanks. They were instructed in the TO condition that the first word presented in each chunk should be written in the leftmost blank, the second word should be written in the center blank, and the third word should be written in the rightmost blank. In the RO condition, subjects were told that for each chunk, the one-syllable word should be written in the leftmost blank, the two-syllable word in the center blank, and the three-syllable word in the right blank. Subjects read the words on the flashcards, either silently for the visual conditions, or aloud for the auditory conditions. After list presentation, subjects immediately began written recall. They were allowed a maximum of 2 min to recall each list, but no subject needed that much time. Subjects could go back at the end of list recall to fill in missing items that they had not recalled in the initial pass through the list. Such items were rare, were marked as such, and are not included in any of the analyses that will be presented in this paper. Each subject received 24 lists altogether, 6 in each of the four major treatment combinations-auditory temporal, visual temporal, auditory reordered, and visual reordered. Subjects were tested individually.

Materials. The materials for this experiment consisted of a set of one-, two-, and three-syllable words chosen from Thorndike and Lorge's (1944) norms to be "A" words (i.e., with a frequency of 50 to 99 per million). Homophones, contractions, and proper nouns were omitted. An attempt was made to avoid very long and very short words.

Design. The experiment was a $2 \times 2 \times 3 \times 3 \times 6$ completely within-subjects, factorial design. The factors were modality (auditory or visual), item-to-item order of recall [temporal order (TO) or reordered (RO) by syllables], chunk (first, second, or third), position within chunk (1, 2, or 3 ), and list (1 to 6 ). For purposes of the analyses that follow, the results were collapsed across lists to result in a number correct (based on six observations) for each of the other four factors in the experiment.

The experiment consisted of 24 separate lists of words that correspond to the 24 trials in which each subject engaged. These lists were divided into four groups of 6 lists each. The four groups of lists were counterbalanced across subjects so that each list was assigned to each major treatment combination-auditory temporal, visual temporal, auditory reordered, visual reordered-an equal number of times. In addition, the order of the four major treatment combinations was counterbalanced so that each of the 24 possible permutations of the order was represented, across subjects, exactly twice (to account for the 48 subjects). The groups of lists, as given above, were randomly assigned with respect to the order of conditions. Within each chunk, over each group of 6 lists, all six of the possible syllable orderings were used. Over the 6 lists in any particular treatment combination, each permutation of order of syllables, with respect to temporal order, was sampled once in each of the three chunks for every subject. The assignment of permutation to list was quasi-random, because the constraint was imposed that the same syllable pattern not occur in more than one chunk within the same list.

\section{Results}

A value of $p>.05$ was chosen as the criterion for significance in the results that are reported below. The data, collapsed across lists, were subjected to a $2 \times 2 \times 3 \times 3$ within-subjects ANOVA.

There was no main effect of modality overall $(F<1)$. There was an effect of order of recall, such that the TO condition gave rise to better performance than did the $\mathrm{RO}$ condition $[\mathrm{F}(1,47)=5.92, \mathrm{MSe}=2.44]$. There was an effect of chunk $[\mathrm{F}(2,94)=99.97, \mathrm{MSe}=6.63]$, such that a bowed-shaped chunk position curve was found. There was an interaction between chunk and order of recall $[\mathrm{F}(2,94)=3.83, \mathrm{MSe}=2.94]$. There was also an interaction of modality and chunk $[\mathrm{F}(2,94)=21.87$, MSe $=3.24]$. This interaction shows a normal modality 
effect: auditory superiority on the last few items (i.e., on the last chunk) and visual superiority on the primacy items (i.e., the first chunk).

Of major importance for the hypothesis was the finding of no tendency whatever for the modality effect to disappear or to reverse when reordering was required. The item-to-item order of recall did not interact with modality $(F<1)$. The triple interaction between modality, order of recall, and chunk was not significant $[F(2,94)=$ $1.18, p=.31]$. Figure 1 shows the interaction between modality and chunk, and the fact that this interaction is obtained regardless of the ordering task.

There were some significant effects with respect to position within chunk (i.e., first item, second item, or third item recalled within a chunk). There was a position main effect $[\mathrm{F}(2,94)=16.67, \mathrm{MSe}=.89]$; an interaction between position and order of recall $[F(2,94)=3.13$, MSe $=.75]$; an interaction between chunk and position $[\mathrm{F}(2,94)=3.91$, $\mathrm{MSe}=.77]$; an interaction between order, chunk, and position $[\mathrm{F}(2,94)=4.52, \mathrm{MSe}=.75]$; and an interaction between modality, chunk, and position $[\mathrm{F}(2,94)=2.43$, MSe $=.59]$. Only the position main effect was a large effect, and only the position main effect was replicated in the experiment that follows. We therefore do not consider the interactions with position any further. There were no other significant effects in this experiment.

\section{Discussion}

The idea that item-to-item directional associations are automatically formed when the input modality is auditory but not visual, and cannot be ignored if they exist, received no support from Experiment 1, which explicitly sought to test this idea. However, it is possible that the experiment did not provide optimal conditions for demonstrat- ing the results of a directional auditory trace, or that the expected obliteration of the modality effect by the reordering task would only show up under circumscribed conditions.

The first possible problem with Experiment 1 was that the condition that was called "auditory" above was a combination of both auditory and visual, because subjects saw the words they read aloud. To test whether this procedure produces results like those found with a tape-recorded pure auditory technique, we ran an experiment comparing the combined condition, a pure auditory condition (presented by means of a prerecorded tape), and a visual condition. Because forward-serial recall is the procedure that is most often used to study modality effects (and since it gives the best results), we used this method of testing.

Subjects were 18 male and female graduate and undergraduate students at the University of British Columbia who were tested individually. The experiment was a $3 \times$ $9 \times 6$ within-subjects factorial design, where the factors were input modality (auditory, auditory + visual, visual), serial position (1-9), and list (1-6). Order of presentation of the three major conditions of interest and list assignment to condition were nuisance factors that were counterbalanced. The three lists used were the first three lists from Experiment 1, and these were rotated through the conditions so that, over subjects, each list occurred six times in the auditory, in the visual, and in the auditory + visual condition. In the auditory condition, the words were presented on tape at a $1.5-\mathrm{sec}$ rate; in the visual condition, the words were shown on flash cards at a $1.5-\mathrm{sec}$ rate; in the auditory + visual condition, the flash cards were read aloud by the subjects. Subjects were told to list the words in specially prepared booklets, writing the first word first, the second word second, and so on down the page. They were told that they could skip

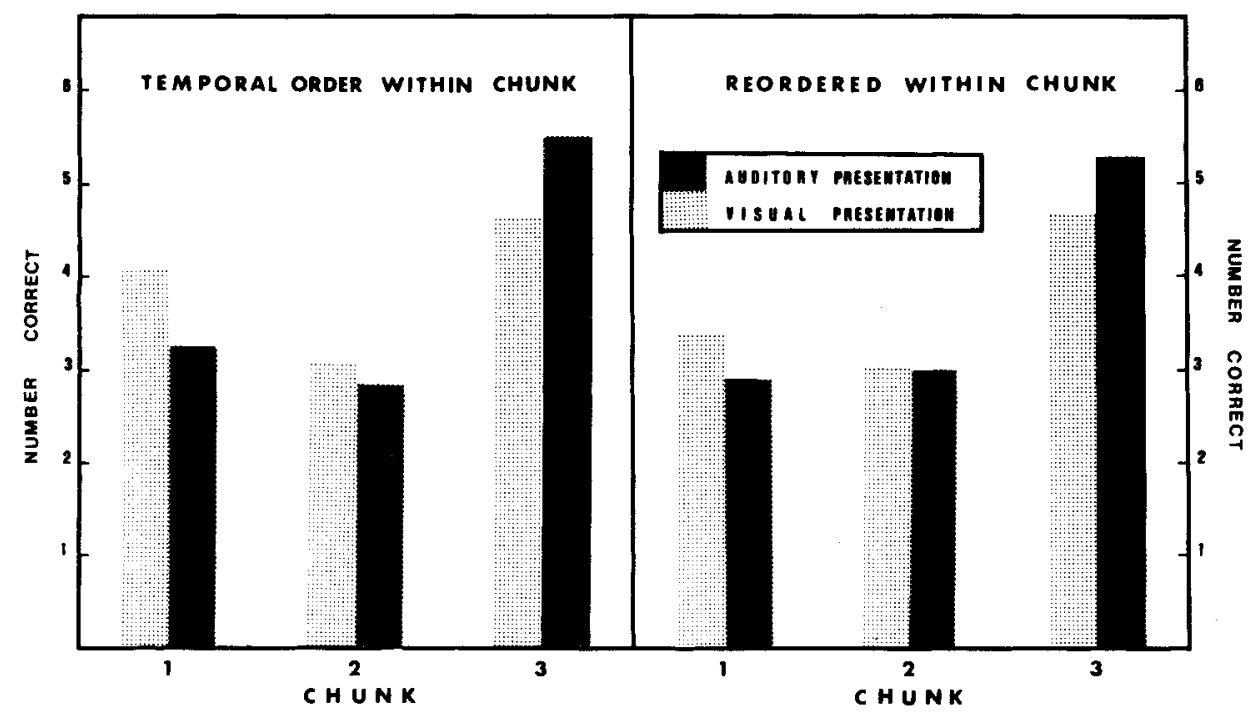

Figure 1. The modality effect in the temporally ordered and the reordered conditions from Experiment 1 . 
down to items later in the list, but they could not go back to fill in earlier items. The experimenter watched to make sure the subjects obeyed the instructions, which they did.

The data were collapsed across lists. The means for each of the three conditions across serial positions are presented in Table 1. An ANOVA showed that there was no main effect of condition $[\mathrm{F}(2,34)=1.11, \mathrm{MSe}=1.37]$. There was an effect of serial position $[F(8,136)=25.10$, MSe $=2.37$. There was also an interaction between condition and serial position $[\mathrm{F}(16,272)=5.38, \mathrm{MSe}=1.57]$. The visual presentation condition showed better serial recall at the beginning of the list and worse recall at the end of the list than did the auditory or the auditory + visual conditions.

Three $t$ tests were conducted on the last three itemsthe recency items - to investigate in a more focal manner the differences in recall in the locus of the modality effect as a function of conditions. The difference in recency between the auditory and the visual conditions (with means respectively of 3.72 and 2.70 ) was significant $[\mathrm{t}(17)=$ 3.95]. Thus, in this experiment a modality effect was found, as expected. The difference between the auditory + visual condition (mean $=3.84$ ) and the visual condition (mean $=2.70$ ) was also significant $[\mathrm{t}(17)=2.99]$. Thus a modality effect also was found with the auditory + visual condition. There was no difference between the auditory (3.72) and the auditory + visual (3.84) conditions [t $(17)=.34]$. The technique of using a spoken-aloud presentation instead of a tape-recorded auditory presentation does not seem to pose any interpretive problems. If anything, the modality effect obtained with the auditory + visual procedure is more extreme than that obtained with the auditory-only method.

The experiment that follows addresses a second point of concern with the procedures used in Experiment 1. Although, in that experiment, the order of recall was controlled on an item-to-item basis within each chunk, the order of recall was not controlled across chunks. It is well known that the delay between study and test or the lag between the input and the output positions of an item can have large effects on the goodness of recall (e.g., Bjork \& Whitten, 1974; Glenberg et al., 1980; Johansson \& Nilsson, 1979). It may have been the case that the output order of the chunks themselves was highly variable or varied systematically depending upon the input modality. Differences in the output order of the chunks could perhaps mask effects that depend upon the item-to-item order manipulation. Alternatively, the recall order of the

Table 1

Mean Frequency of Recall (out of 6) for the Auditory, Auditory + Visual, and Visual Conditions as a Function of Input Serial Position

\begin{tabular}{lccccccccc}
\hline & \multicolumn{8}{c}{ Serial Position } \\
\cline { 2 - 10 } Condition & 1 & 2 & 3 & 4 & 5 & 6 & 7 & 8 & 9 \\
\hline Auditory & 4.44 & 3.17 & 3.11 & 1.72 & 1.67 & 1.44 & 2.44 & 3.11 & 5.61 \\
Auditory & & & & & & & & & \\
+ Visual & 3.78 & 2.56 & 2.44 & 1.61 & 1.44 & 2.28 & 3.17 & 3.56 & 4.78 \\
Visual & 5.28 & 4.22 & 3.28 & 2.28 & 1.94 & 2.22 & 2.22 & 2.72 & 3.17 \\
\hline
\end{tabular}

chunks might be of major importance for the modality effect, accounting for many of the findings that were cited in the introduction. In Experiment 2, the chunk order of recall and the item-to-item order of recall are manipulated.

\section{EXPERIMENT 2}

\section{Method}

The design of the experiment was like that of Experiment 1, except that there was one additional between-subjects factor: chunk order [either 1,2, 3 (forward) or 3, 2, 1 (backward)]. Instead of using two replications of the entire counterbalanced design, as had been the case in Experiment 1, one replication was conducted in each of the chunk-order conditions. Forty-eight subjects participated in the experiment. The subjects were University of British Columbia students who were paid $\$ 4$ for participating in the 1-h session. Subjects were tested individually.

The experimenter watched each subject as he or she wrote down recall of each word. In the forward-order task, subjects were told to write the first three words on the top line, the second three words on the second line, and the third three words on the bottom line. Similarly, in the backward-order task, subjects were instructed to write recall of the last chunk on the third line, the second chunk on the second line, and the first chunk on the top line. The experimenter watched as the subject wrote down each word, and circled any discrepancies from the required order. Items recalled out of order are not considered in the analyses that follow. There were very few such cases, however.

\section{Results}

The major results of interest can be seen by examining Figure 2. There was a main effect of chunk order $[F(1,46)$ $=13.75, \mathrm{MSe}=578.5]$. The backward-order conditions shown in the top two panels of the figure produced higher recall than the forward-order conditions. There was a main effect of item-to-item recall order, such that the TO condition was better than the RO condition $[F(1,46)$ $=11.89, \mathrm{MSe}=143.3 \mathrm{~J}$. In the figure, the left panels show better overall performance than the right panels. There was an interaction between modality and chunk $[F(2,92)=18.6, \mathrm{MSe}=2.95]$. This interaction indicates that the usual modality effect was found in this experiment: auditory recall was better than visual in the last chunk, and visual was better than auditory on the first chunk. There was an interaction between chunk-order of recall and chunk such that the first chunk was recalled better in the forward order, and the last chunk was recalled better if recall was in the backward order $[F(2,92)=$ $50.58, \mathrm{MSe}=6.28 \mathrm{]}$. These results were all expected and offer little new information. They do suggest that there were no major unanticipated problems in the experiment.

There was a significant triple interaction among chunkorder, modality, and chunk $[\mathrm{F}(2,92)=10.77$, MSe $=$ 2.95]. As can be seen from the bottom two panels of Figure 2, recall in the forward order for the visual conditions decreased monotonically over chunks. There was no recency effect if the input modality was visual. There was, however, a large recency effect in the auditory modality. The difference between the auditory and visual conditions on the last chunk (i.e., the modality effect) is pronounced when recall was in the forward order. In con- 


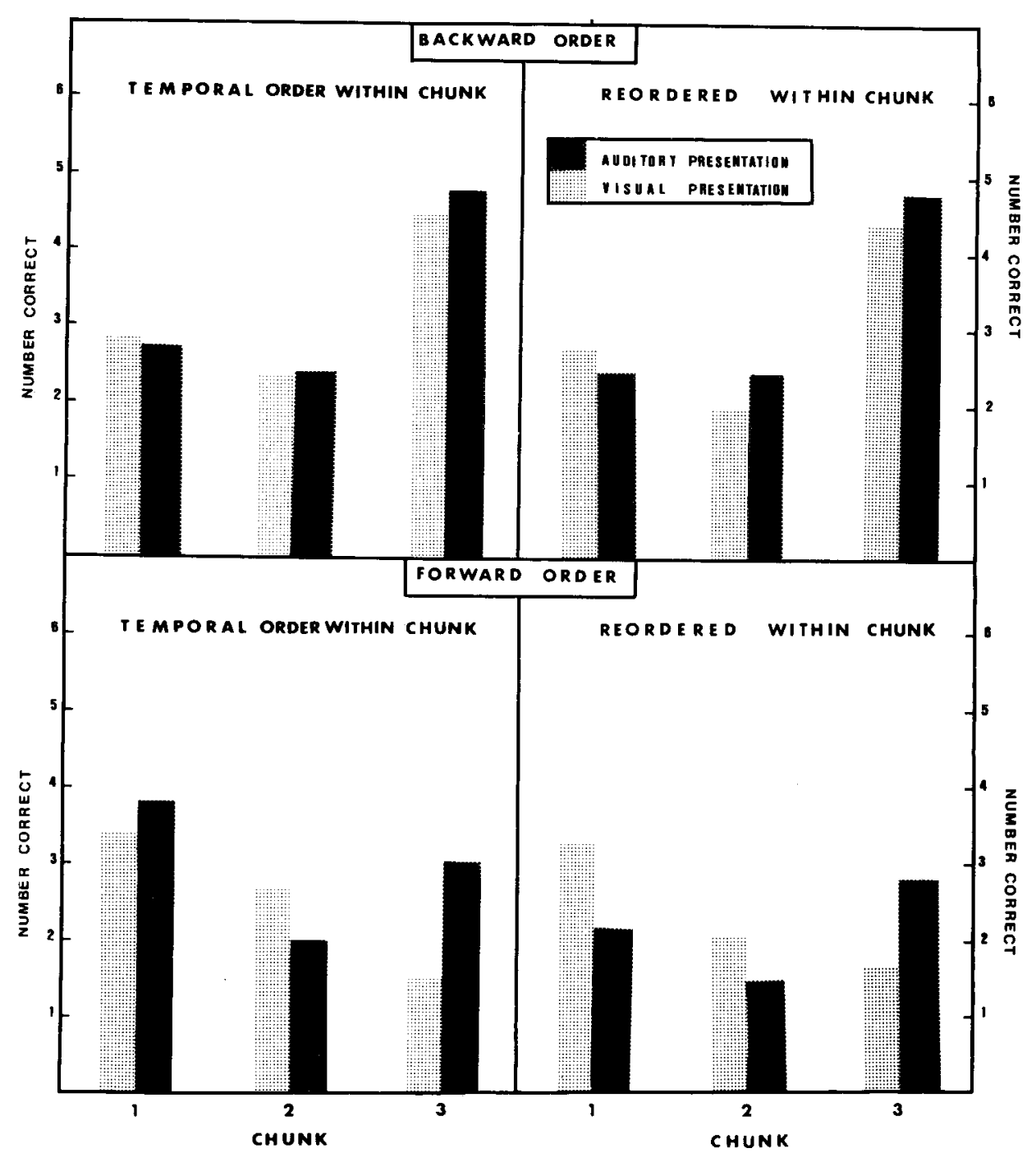

Figure 2. The modality effect in the temporally ordered and reordered conditions from Experiment 2 . The top two panels show the effect when the chunks were recalled in the backward order, and the bottom two panels show the effect when recall was in a forward order by chunk.

trast, the top panels show that, when recall was in a backward order by chunks, both the auditory and the visual conditions showed a recency effect, and there was not much of a modality effect. This is the most impressive result in this experiment. We will discuss it further in the section that follows.

There was a significant triple interaction among chunk order, modality, and item-to-item order of recall $[\mathrm{F}(1,46)$ $=5.28, \mathrm{MSe}=86.49]$. In the backward-chunk order there was little modality-specific difference regardless of whether the TO or the RO item-to-item order of recall was required. In the forward-chunk order, however, auditory superiority was found in the TO condition, whereas a slight visual superiority was found in the RO condition. The directional trace hypothesis implies not only that there should be an interaction between modality and item-toitem order of recall, but also that the effects of this inter- action should be felt particularly on the last few items or the last chunk, where the modality effect is normally located. The modality $\times$ item-to-item order $\times$ chunk interaction was indeed significant $[F(2,92)=4.29$, MSe $=2.98 \mathrm{~J}$. However, the pattern of this interaction is inconsistent with the directional trace hypothesis. In particular, the RO condition did not eliminate the modality effect (i.e., the auditory superiority at the end of the list). Instead, the RO condition resulted in auditory inferiority at the beginning of the list or in the first chunk. This triple interaction also qualifies a double interaction between item-to-item order and chunk $[\mathrm{F}(2,92)=3.84, \mathrm{MSe}=$ 2.59 ], showing that the item-to-item reordering manipulation had its detrimental effect on the first two chunks and not on the last chunk.

There was an effect of position within chunk $[F(2,92)$ $=3.0$, MSe $=1.55]$, showing that the first or the one- 
syllable word in each chunk was recalled slightly better than the other two items within each chunk. There were no interactions with position within chunk, and there were no other significant effects in Experiment 2.

\section{Discussion}

The two results that were noteworthy in this experiment were the findings that (1) the item-to-item reordering manipulation did not affect recall in the recency part of the curve, but rather affected the primacy part of the curve, and (2) the order of recall by chunks had a dramatic effect on the modality effect. Each of these two results will be discussed in turn.

The fact that the item-to-item reordering manipulation failed to affect performance in the recency part of the serial-position curve indicates that the modality effect is probably not attributable to a directional auditory trace. Had there been a directional auditory trace (especially for the last few list items), then auditory recall should have been inhibited when subjects had to do a task that necessitated that they work against the natural order of that trace. This was not found to be the case in either of the experiments presented here. The auditory superiority on the last chunk was unaffected by the item-to-item reordering manipulation.

As well as showing this negative effect-that the modality effect seems not to be attributable to a directional auditory trace-the present experiment provided some positive hints concerning the locus of the modality effect. One factor that did have a major effect on the difference between auditory and visual recall in the last chunk was the order of output of the chunks themselves. When recall was in a backward order by chunk, the magnitude of the modality effect was found to be much diminished as compared to when recall was in a forward order by chunk. This result is, of course, consistent with the results of Madigan (1971), who found a modality effect when recall was in a forward-serial order but not when it was in a backward-serial order. The present results qualify the earlier results by indicating that the main locus of this difference, depending upon output order, may be at the level of the higher order units, or chunk access, rather than at the level of the items within chunks.

Input modality has been used as one of the many components of events that have been changed when environmental context effects have been studied (Geiselman \& Bjork, 1980; Smith, Glenberg, \& Bjork, 1978). One question of concern is whether context has its effect at the item level or at the level of higher order units or chunks. Context effects have been found primarily at the level of the higher order units in drug context studies (Eich, 1980), and at both the item level and that of higher order units in environmental context studies (Smith et al., 1978). The main results of the present studies indicate that one contextual variable, modality, has its biggest effects primarily at the level of the higher order units. In order to further investigate, in a more fine-grained manner, the influence of modality on higher order unit accessibility, as compared to the retrieval of the items contained within the higher order units, an additional analysis was conducted on the last (modality effect) chunk in Experiment 2 .

\section{Additional Analyses}

Following the analysis used by Eich and Birnbaum (1982), the data from the third chunk (collapsed across lists and across the item-to-item ordering) were used to compute the probability of chunk access. Chunk access was defined as recall of at least one item from a given chunk. Since there were 12 auditory lists and 12 visual lists per subject in the experiment, the chunk-access scores were proportions based on 12 observations for each modality. The chunk-access scores were submitted to a $2 \times$ 2 ANOVA, in which the factors were chunk order and modality. As the left panel of Table 2 shows, in the backward-order chunk, access in both modalities was very good, whereas there was a $30 \%$ difference in chunk access favoring the auditory modality when recall was in the forward order. The main effect of modality was significant $[\mathrm{F}(1,46)=59.72, \mathrm{MSe}=.02]$; the main effect of chunk order was significant $[F(1,46)=94.86$, MSe $=.05]$; and the interaction between modality and chunk order was significant $[\mathrm{F}(1,46)=39.69$, MSe $=.02]$. It is difficult to interpret the interaction because it could be due to a ceiling effect in the backward-order condition. The main result to note is the size of the modality effect when the dependent measure is chunk access: about a $30 \%$ difference favoring auditory presentation when recall was in a forward order.

A second analysis was conducted, this time looking at the probability of recall given that a chunk was accessed. As can be seen from the right panel of Table 2, there is a small, but significant $[\mathrm{F}(1,46)=12.78, \mathrm{MSe}=.02]$, advantage for the auditory modality. The effect of chunk order was significant $[\mathrm{F}(1,46)=178.18, \mathrm{MSe}=.02]$. The interaction between modality and chunk order was not significant. The fact that the within-chunk measure did not change, even when the output interference was increased (in the forward-order condition) and when the modality effect itself was greatly magnified, indicates that the influence of output order on the modality effect probably has little to do with accessibility of the nested items. Instead, the accessibility of larger chunks seems most important. Both the main results of the experiment and the additional analysis point to this conclusion.

Table 2

The Probability of Chunk Retrieval and Within-Chunk Retrieval in the Last Chunk of Experiment 2

\begin{tabular}{cccccc} 
& \multicolumn{2}{c}{ Chunk Retrieval } & & \multicolumn{2}{c}{ Within-Chunk Retrieval } \\
\cline { 2 - 3 } \cline { 5 - 6 } Modality & $\begin{array}{c}\text { Forward } \\
\text { Order }\end{array}$ & $\begin{array}{c}\text { Backward } \\
\text { Order }\end{array}$ & & $\begin{array}{c}\text { Forward } \\
\text { Order }\end{array}$ & $\begin{array}{c}\text { Backward } \\
\text { Order }\end{array}$ \\
\hline Auditory & .81 & 1.00 & & .69 & .96 \\
Visual & .51 & .97 & .59 & .92 \\
\hline
\end{tabular}




\section{GENERAL DISCUSSION}

The relation between output order and the modality effect has posed a puzzle for the last 10 years. The present results indicate that one possible solution to that puzzlethe directional auditory trace hypothesis-is not, in fact, the solution. Input modality appears to have its major effect at a level of organization higher than the level of horizontal associations between particular temporally contiguous items that is implied by the directional trace hypothesis. While the present results contradict the directional trace hypothesis, they are not in direct conflict with other theoretical explanations of the modality effect, such as the precategorical acoustic storage (PAS) model of Crowder and Morton (1969), the lateral inhibition model of Crowder (1978), some versions of the functional view of Nilsson (1979) or of Ronnberg, Archer, and Ohlsson (1980), the two-store view of Murdock and Walker (1969), and the synthesis view of Ronnberg and Ohisson (1980). While these ideas about the modality effect are not directly contradicted by the present results, they also provide little insight into why auditory superiority is found at the end of the list and visual superiority is typically found at the beginning of the list. Still less do they suggest why output order at the level of the chunks should interact with the input modality. The idea that there is differential susceptibility to interference in the auditory and visual modalities, as has been proposed by Nilsson et al. (1979), may be elaborated to suggest why there are modality-specific differences in the level of recall depending upon output order, and to suggest why the serial position curves might show the particular pattern that is typically found. It is clear, though, that further theoretical investigation is necessary to consolidate our understanding of modality effects in human memory.

\section{REFERENCES}

Bjork, R. A.. \& Whitten, W. B. (1974). Recency-sensitive retrieval processes in long-term free recall. Cognitive Psychology, 6, 173-189. CRAIK, F. I. M. (1969). Modality effects in short-term storage. Journal of Verbal Learning \& Verbal Behavior, 8, 658-664.

Crowder, R. G. (1978). Mechanisms of auditory backward masking in the stimulus suffix effect. Psychological Review, 85, 502-524.
Crowder, R. G., \& Morton, J. (1969). Precategorical acoustic storage (PAS). Perception \& Psychophysics, 5, 365-373.

DREWNOWSKI, A. (1980). Memory functions for vowels and consonants: A reinterpretation of acoustic similarity effects. Journal of Verbal Learning \& Verbal Behavior, 19, 176-193.

EICH, E. \& Birnbaum, I. M. (1982). Repetition, cuing, and statedependent memory. Memory \& Cognition, 10, 103-114.

EICH, J. E. (1980). The cue-dependent nature of state dependent retrieval. Memory \& Cognition, 8, 157-173.

Geiselman, R. E., \& BjoRK, R. A. (1980). Primary versus secondary rehearsal in imagined voices: Differential effects on recognition. Cognitive Psychology, 12, 188-205.

Glenberg, A. M., Bradley, M. M., Stevenson, J. A., Kraus, T. A., TKachuk, M. J., Gretz, A. L., Fish, J. L., \& Turpin, B. M. (1980). A two-process account of long-term serial position effects. Journal of Experimental Psychology: Human Learning \& Memory, 6, 355-369.

JoHANSSON, B. S., \& NILSSON, L.-G. (1979). Boundary conditions for the modality effects: Type of material and time of test. Scandinavian Journal of Psychology, 20, 27-35.

MADIGAN, S. A. (1971). Modality and recall order interactions in shortterm memory for serial order. Journal of Experimental Psychology, 87, 294-296.

Metcalfe, J., Glavanov, D., Murdock, M. (1981). Spatial and temporal processing in the auditory and visual modalities. Memory \& Cognition, 9, 351-359.

Murdock, B. B., JR., \& Walker, K. D. (1969). Modality effects in free recall. Journal of Verbal Learning \& Verbal Behavior, 8, 665-676.

Nilsson, L. -G. (1979). A functional interpretation of the modality effect. Scandinavian Journal of Psychology, 20, 259-265.

Nilsson, L.-G., WRight, E., \& Murdock, B. B., JR. (1975). The effects of visual presentation method on single-trial free recall. Memory \& Cognition, 3, 427-433.

Nilsson, L.-G., Wright, E., \& Murdock, B. B., JR. (1979). Order of recall, output interference, and the modality effect. Psychological Research. 41, 63-78.

PENNEY, C. G. (1975). Modality effects in short-term verbal memory. Psychological Bulletin, 82, 68-84.

Ronnberg, J., Archer, T., \& Ohlsson, K. (1980). Temporal factors in audition and vision: A functional emphasis. Scandinavian Journal of Psychology, 21, 241-247.

Ronnberg, J., \& Ohlsson, K. (1980). Channel capacity and processing of modality specific information. Acta Psychologica, 44, 253-267.

SMith, S. M., GlenberG, A., \& Biork, R. A. (1978). Environmental context and human memory. Memory \& Cognition, 6, 342-353.

THORNDike, E. L., \& LoRGE, I. (1944). The teacher's word book of 30,000 words. New York: Bureau of Publications, Teacher's College, Columbia University

(Manuscript received October 18, 1984; revision accepted for publication July 18,1985 .) 